\title{
ON THE ZEROS OF A POLYNOMIAL AND ITS DERIVATIVE
}

\author{
A. W. GOODMAN, Q. I. RAHMAN AND J. S. RATTI
}

1. Introduction. The Gauss-Lucas Theorem states that if $S$ is the set of zeros of the polynomial

$$
P(z)=\prod_{k=1}^{n}\left(z-z_{k}\right)
$$

then every zero of the derivative $P^{\prime}(z)$ is contained in the smallest convex set that contains $S$. This theorem has been rather thoroughly investigated [2] and sharpened in several ways. However, there is one related question that deserves attention, namely given one specific zero $z_{z}$ of $P(z)$, what can be said about a neighborhood of $z_{z}$ that will always contain a zero of $P^{\prime}(z)$ ?

By a translation, followed by a stretching (or shrinking), we may require that all the zeros of $P(z)$ are in $\varepsilon$, the closed unit disk. Further by a rotation we may set $z_{n}=a$, where $0 \leqq a \leqq 1$. With this normalization the problem can now be formulated precisely.

Let $P(a, n)$ be the set of all $n$th degree polynomials $P(z)$ that have all of their zeros in $\varepsilon$, and at least one zero at $z=a, 0 \leqq a \leqq 1$. Let $D(a, n)$ be a minimal region with the property that if $P(z) \in \mathcal{P}(a, n)$, then $P^{\prime}(z)$ has at least one zero in $D(a, n)$. Describe $D(a, n)$.

This problem seems to be rather difficult. As far as the authors are aware, the first step in the direction of this problem is contained in the conjecture due to Iliev $[1$, p. 25]. Let $g(a)$ be the intersection of the disk $|z-a| \leqq 1$ with $\mathcal{E}$. Then, according to Iliev, $\mathscr{g}(a)$ always contains at least one zero of $P^{\prime}(s)$.

In this paper we replace $g(a)$ by a region $\mathcal{C}^{*}(a)$ which is in general much smaller, and we conjecture that every $P(z)$ in $P(a, n)$, has at least one root in $e^{*}(a)$. We can prove this proposition if $a=1$. The case $a=0$ is trivial. For $0<a<1$, the conjecture is still open.

2. The main result. Let $e^{*}(a)$ be the closed disk, with center at $z=a / 2$ and radius $1-a / 2$, where $0 \leqq a \leqq 1$. In particular if $a-1$, this is the disk with center at $1 / 2$ and radius $1 / 2$.

TheORem. Let $P(8)$ be a polynomial with all its seros in $\varepsilon$, and at least one zero at $s_{n}=1$. Then $P^{\prime}(s)$ has at least one zero in $e^{*}(1)$. Further if $e^{*}(1)$ is replaced by any open subset of $C^{*}(1)$, then the assertion is false.

Presented to the Society, January 24, 1968; received by the editors June 28, 1968. 
Proof. If $z=1$ is a multiple root of $P(z)$ then $z=1$ is also a root of $P^{\prime}(z)$ and since 1 is in $e^{*}(1)$, the assertion is true in this case. Henceforth we assume that $z=1$ is a simple zero. Let $P(z)$ be given by (1.1). Then

$$
\phi(z) \equiv \frac{P^{\prime}(z)}{P(z)}=\frac{1}{z-1}+\sum_{k=1}^{n-1} \frac{1}{z-z_{k}} .
$$

We map $\varepsilon$ onto the right halfplane by setting $w=(1+z) /(1-z)$. This transformation takes $\phi(z)$ into

$$
\phi\left(\frac{w-1}{w+1}\right) \equiv \Phi(w)=\frac{w+1}{2}\left[-1+\sum_{k=1}^{n-1} \frac{w_{k}+1}{w-w_{k}}\right],
$$

$w_{k}=\left(1+z_{k}\right) /\left(1-z_{k}\right), k=1,2, \cdots, n-1$. By hypothesis $\left|z_{k}\right| \leqq 1$, and hence $R\left(w_{k}\right) \geqq 0$, for $k=1,2, \cdots, n-1$. If $\Phi(w)$ has a zero at $w=\eta$, then $\phi(z)$ will have a zero at the image point $\zeta=(\eta-1) /(\eta+1)$, and hence $P^{\prime}(\zeta)$ will also be zero. We multiply the last factor in (2.2) by $\Pi\left(w-w_{k}\right)$ and examine the resulting polynomial equation.

$$
w^{n-1}-(n-1+2 \sigma) w^{n-2}+\cdots=0,
$$

where $\sigma \equiv w_{1}+w_{2}+\cdots+w_{n-1}$. If $\eta_{1}, \eta_{2}, \cdots, \eta_{n-1}$ are the roots of (2.3), then

$$
R\left(\eta_{1}+\cdots+\eta_{n-1}\right)=R(n-1+2 \sigma) \geqq n-1 .
$$

Hence equation (2.3) has at least one root $\eta^{*}$ such that $R\left(\eta^{*}\right) \geqq 1$. Then $\zeta^{*} \equiv\left(\eta^{*}-1\right) /\left(\eta^{*}+1\right)$ is a zero of $P^{\prime}(z)$ and $\zeta^{*}$ lies in the disk $e^{*}(1)$. Consider now the particular polynomial

$$
\begin{aligned}
P_{3}(z) & =(z-1)\left(z-e^{i \alpha}\right)\left(z-e^{-i \alpha}\right) \\
& =z^{3}-(1+2 \cos \alpha) z^{2}+(1+2 \cos \alpha) z-1 .
\end{aligned}
$$

Then $P_{3}^{\prime}(z)$ has zeros at

$$
\zeta_{1}, \zeta_{2}=(1 / 3)\left(1+2 \cos \alpha \pm i(2(1+2 \cos \alpha)(1-\cos \alpha))^{1 / 2}\right),
$$

where the quantity under the radical is positive if $0<\alpha<2 \pi / 3$. It is an easy matter to show that as $\alpha$ runs from 0 to $2 \pi / 3$ the points $\zeta_{1}$ and $\zeta_{2}$ describe the boundary of $\mathfrak{C}^{*}(1)$.

\section{REFERENCES}

1. W. K. Hayman, Research problems in function theory, Athlone Press, University of London, 1967.

2. M. Marden, The geometry of the zeros of a polynomial, Math. Surveys, No. 3, Amer. Math. Soc., Providence, R. I., 1949.

University OF SOUth Florida 